\title{
Energy acceptance of the St. George recoil separator
}

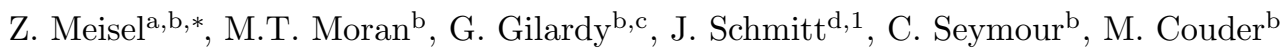 \\ ${ }^{a}$ Institute of Nuclear 83 Particle Physics, Department of Physics \& Astronomy, Ohio University, Athens, OH 45701, USA \\ ${ }^{b}$ Department of Physics, Joint Institute for Nuclear Astrophysics, University of Notre Dame, Notre Dame, IN 46556, USA \\ ${ }^{c}$ Centre d'Études Nucléaires de Bordeaux Gradignan, UMR 5797 CNRS/IN2P3 - Université de Bordeaux, 19 Chemin du Solarium, CS \\ 10120, F-33175 Gradignan, France \\ ${ }^{d}$ Department of Physics \& Astronomy, Clemson University, Clemson, SC 29634, USA
}

\begin{abstract}
Radiative alpha-capture, $(\alpha, \gamma)$, reactions play a critical role in nucleosynthesis and nuclear energy generation in a variety of astrophysical environments. The St. George recoil separator at the University of Notre Dame's Nuclear Science Laboratory was developed to measure $(\alpha, \gamma)$ reactions in inverse kinematics via recoil detection in order to obtain nuclear reaction cross sections at the low energies of astrophysical interest, while avoiding the $\gamma$-background that plagues traditional measurement techniques. Due to the $\gamma$ ray produced by the nuclear reaction at the target location, recoil nuclei are produced with a variety of energies and angles, all of which must be accepted by St. George in order to accurately determine the reaction cross section. We demonstrate the energy acceptance of the St. George recoil separator using primary beams of helium, hydrogen, neon, and oxygen, spanning the magnetic and electric rigidity phase space populated by recoils of anticipated $(\alpha, \gamma)$ reaction measurements. We find the performance of St. George meets the design specifications, demonstrating its suitability for $(\alpha, \gamma)$ reaction measurements of astrophysical interest.
\end{abstract}

Keywords:

Recoil mass separator; Radiative alpha-capture;

PACS: 26.20.Fj, 29.30.Aj

\section{Introduction}

Precise $(\alpha, \gamma)$ reaction cross sections are crucial to ac- ${ }^{23}$ curately model a variety of astrophysical phenomena [1], ${ }_{25}^{24}$ such as the production of the $s$-process neutron-source ${ }_{26}$ nucleus ${ }^{22} \mathrm{Ne}[2]$, the carbon-to-oxygen abundance ratio ${ }_{27}^{26}$ of stellar cores [3], nucleosynthesis in massive stars [4], ${ }_{28}^{27}$ the abundance of ${ }^{44} \mathrm{Ti}$ produced in core-collapse supernovae [5], and the nuclear reaction sequence occurring in ${ }_{30}^{29}$ type-I x-ray bursts [6]. Owing to the relatively low kinetic ${ }_{31}^{30}$ energies of nuclei in the relevant astrophysical conditions, ${ }_{32}$ traditional nuclear reaction measurements in which the outgoing $\gamma$ ray is measured often suffer from prohibitively ${ }_{34}^{33}$ high $\gamma$-backgrounds and, for high $Q$-value reactions, complicated $\gamma$-ray cascades [7]. One approach which has been adopted to overcome this difficulty employs inverse kine- ${ }_{37}^{36}$ matics and a recoil separator, in which the nuclear recoil ${ }_{38}^{37}$ produced in the $(\alpha, \gamma)$ reaction is electromagnetically separated from unreacted beam nuclei on the basis of their mass differences and identified with various combinations ${ }_{41}^{40}$ of time-of-flight and energy-loss measurements [8]. Recoil- ${ }_{42}^{41}$ $\gamma$ coincidences substantially improve the signal-to-noise ${ }_{43}^{42}$

\footnotetext{
* Corresponding author

Email addresses: meisel@ohio.edu (Z. Meisel), mcouder@nd.edu (M. Couder)

${ }^{1}$ Present Address: Department of Physics and Astronomy, Michi- ${ }^{47}$ gan State University, East Lansing, MI 48824, USA
}

compared to traditional techniques and provide significantly reduced cross section uncertainties [9].

In order to undertake $(\alpha, \gamma)$ reaction cross section studies at astrophysically relevant energies for nuclei with nuclear mass $A \leq 40$, the St. George recoil separator at the University of Notre Dame's Nuclear Science Laboratory (NSL) has been developed [10]. The recoil separator technique generally relies on using inverse kinematics, where a heavy ion beam is impinged on a lighter nuclear target; for $(\alpha, \gamma)$ reactions with St. George, the HIPPO helium gas-jet produces the desired target $[11,12]$. Unreacted incident beam particles and nuclear reaction recoils both exit the gas-jet with similar momenta. For a typical cross section of $1 \mu \mathrm{b}$ and a gas-jet density of $10^{17}$ atoms $/ \mathrm{cm}^{2}$, the ratio for recoils to unreacted beam nuclei is 1 part in $10^{13}$. In order to be effective, a recoil separator must accept all (or most [13]) of the nuclear reaction recoils and have a mass separation sufficient to produce a recoil/beam ratio allowing for practicable particle identification.

Due to the prompt $\gamma$-ray emission, nuclear reaction recoils leave the target with a range of energies and angles, according to the reaction $Q$-value, $\gamma$-ray cascade, and $\gamma$ emission angles [8]. For St. George, the 'acceptance' is the angle-energy phase space of nuclear reaction recoils that the separator transmits to the recoil detection-plane with $100 \%$ efficiency. Accurate cross section measurements with the recoil separator technique rely on a thorough charac- 
terization of the recoil separator acceptance.

In a crucial first step of the St. George recoil separa-100 tor commissioning process, we have determined the energy 101 acceptance of St. George for a range of beam species, span-102 ning the magnetic and electric rigidity phase space antic-103 ipated for recoils of future $(\alpha, \gamma)$ cross section measure-104 ments. We discuss the experimental set-up in Section 2,105 our energy acceptance measurement method in Section 3,106 and the measurement results and discussion thereof in Sec-107 tion 4, prior to summarizing our work in Section 5.

\section{Experimental set-up}

Stable ion beams are delivered to St. George by the ${ }^{112}$ recently installed $5 \mathrm{MV}$ single-ended Pelletron accelerator ${ }^{113}$ St. Ana, from National Electrostatics Corporation ${ }^{2}$, at the ${ }^{114}$ NSL. Ion beams produced by a Nanogan ECR ion source, ${ }^{115}$ from Pantechnik ${ }^{3}$, are accelerated with St. Ana, energy-116 selected by a $90^{\circ}$ analyzing magnet, and transported down ${ }^{117}$ $\mathrm{a} \approx 22 \mathrm{~m}$ beam-line, which contains several quadrupole and ${ }^{118}$ steerer magnets, to St. George with beam intensities up- ${ }^{119}$ wards of $100 \mu \mathrm{A}$. The configuration of the transport line $\mathrm{e}^{120}$ at the time of this experiment, shown in Figure 1, con- ${ }^{121}$ sisted of the horizontal $\mathrm{X}$ and vertical $\mathrm{Y}$ steerer magnet ${ }^{122}$ pair $\mathrm{XY}_{1}$ and $\mathrm{XY}_{2}$ following the accelerator momentum- ${ }^{123}$ analyzing magnet and preceding the quadrupole doublet ${ }^{124}$ $\mathrm{QD}_{1}$, followed by said quadrupole doublet, steerer pair ${ }^{125}$ $\mathrm{XY}_{3}$ and $\mathrm{XY}_{4}$, the second quadrupole doublet $\mathrm{QD}_{2}$, the $\mathrm{e}^{126}$ single steerer $\mathrm{XY}_{5}$, a dipole 'switching' magnet, and (not ${ }^{127}$ shown) an XY steerer and a quadrupole triplet. Beam po- ${ }^{128}$ sition monitors, Faraday cups, and fluorescing quartz windows are employed at various locations along the transport line to provide diagnostic information during beam tuning.

St. George consists of eighteen ion optical elements: six ${ }_{130}$ dipole magnets, eleven quadrupole magnets (four doublets131 and one triplet), and a Wien filter, as shown in Figure 2.132 The last quadrupole, Q11, is followed by an ion detection 133 chamber in which ions can be identified via total energy $y_{134}$ loss and time-of-flight. Several ports are available along ${ }_{135}$ the St. George beam line and on the magnets for diag-136 nostic equipment to assess the properties of transmitted ${ }_{137}$ ions. For future $(\alpha, \gamma)$ reaction measurements, St. George ${ }_{138}$ will be preceded by the HIPPO gas-jet [11], which will ${ }_{139}$ serve as a relatively uniform high-density reaction tar-140 get [12]. For the acceptance measurements presented here,141 the primary beam from St. Ana was transmitted directly ${ }_{142}$ into St. George, where two $2 \mathrm{~mm}$ diameter collimators 143 $20 \mathrm{~cm}$ apart were employed just prior to the entrance of ${ }_{144}$ St. George to ensure the beam entered St. George on-axis.145 The collimators had the added benefit of limiting beam ${ }_{146}$ currents to a few $\mu A$ in order to protect diagnostic equip-147 ment.

\footnotetext{
${ }^{2}$ http://www.pelletron.com

${ }^{3}$ http://www.pantechnik.com
}

Diagnostic equipment along St. George includes Faraday cups, fluorescing quartz windows, and electrically insulated slits. Faraday cups, used to monitor beam transmission, are located following the first, second, and fifth dipole magnets, the Wien filter, and the eleventh quadrupole magnet. Each Faraday cup is equipped with a $-300 \mathrm{~V}$ secondary electron suppression electrode to ensure reliable current readings. Electrically insulated slits with current readouts, used for the energy acceptance measurements to monitor the beam width and centering, are located before and after each dipole magnet, as well as $1.59 \mathrm{~m}$ downstream of the Wien filter exit. These slits will be used in future $(\alpha, \gamma)$ reaction measurements to improve beamrejection properties of St. George. In particular, the slits following the Wien filter, referred to as the 'mass slits', are located at the position optimized for beam-rejection, which provides a horizontal achromatic focus for recoil nuclei. The slits and Faraday cups each have a lower-limit for their current sensitivity of $\approx 1 \mathrm{pA}$. The $0^{\circ}$ exit port of the first, third, and fifth dipole magnets, as well as the exit port of the recoil detection chamber, are equipped with fluorescing quartz windows from McMaster-Carr ${ }^{4}$, outside of which (at atmosphere) is a camera. The quartz windows are used to assess the beam shape at their respective locations and, more importantly, are used to determine if the beam is being transported along the ion-optical center of the quadrupole magnets preceding that quartz. The quartz viewers also serve as a crude way to determine beam transmission, as their fluorescence is visible at relatively low beam currents.

\section{Energy acceptance measurement method}

In inverse kinematics, the products of an $(\alpha, \gamma)$ reaction are emitted from the target position with the same average momentum as the unreacted beam in a narrow cone, where the opening angle is primarily determined by the reaction $Q$-value and center-of-mass energy (See Equation 13 of Reference [8].). St. George is designed to accept and transmit all recoil nuclei of a single charge-state emitted from the target with a maximum angle of $\pm 40 \mathrm{mrad}$ and energy spread from the central recoil energy of $\pm 8 \%$ [10]. The maximum recoil energy deviations occur when $\gamma$ rays are emitted at $0^{\circ}$ or $180^{\circ}$ in the center of mass.

In this study, we mimicked the recoil energy spread by employing a beam in the absence of a target in order to verify that, at $0^{\circ}$ (i.e. for no angular deflection), $100 \%$ of ions within $\pm 8 \%$ of the energy for which the separator was tuned were transmitted to the end of St. George. A similar technique was employed to determine the energy acceptance of the ERNA recoil separator [14, 15]. Transmission through the recoil separator was determined by comparing beam current measurements provided by the

\footnotetext{
${ }^{4}$ http://www.mcmaster.com
} 


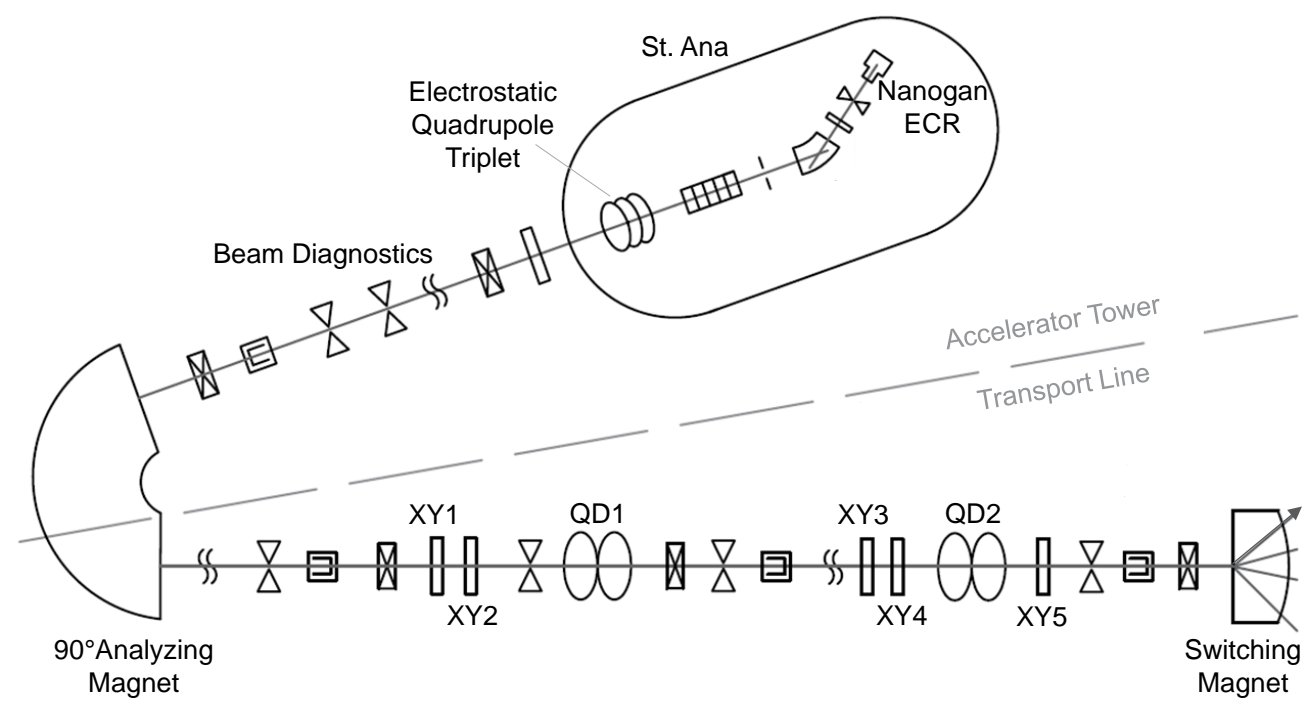

(See Figure 1.) were adjusted to deliver an ion beam of the chosen species and energy with a current of several hundred nA to the St. George target location. The accelerator and transport line elements were adjusted to send beam through the two collimators at the target location.

1.2. With no field in the B1 dipole magnet ${ }^{7}$, the beam was impinged on the B1 $0^{\circ}$ quartz viewer. The Q1 and Q2 quadrupole magnets of St. George separately had their field strengths adjusted from the minimum to $>50 \%$ of the maximum field strength. The image of the beam-spot on the B1 $0^{\circ}$ exit-port fluorescingquartz was monitored for shifts in the spot centroid. Any deviation from a 'non-steering' beam, shown in Figure 3, was corrected by adjusting the last few steering magnets of the St. Ana transport line.

1.3. The B1 and B2 dipole magnets were adjusted to their nominal fields for the species of interest at the tune-energy, sending the beam to the $\mathrm{B} 30^{\circ}$ exit-port quartz-viewer, with no field on the B3 dipole. The correction process of the previous step was repeated. Though this step is seemingly redundant, the longer distance of travel of the beam from Q1 and Q2 to this quartz provided a higher sensitivity to the presence of steering in the ion optics. This step could not be repeated for the $0^{\circ}$ exit port of the B5 dipole magnet since the ion beam was too defocused at that location without using quadrupole magnets Q3-Q9, a necessary condition to test for beam steering.

${ }^{7}$ We note that residual magnetic fields on the dipoles do not impact our methods, as the effect would only be a horizontal translation of the beam spot on a quartz viewer. 


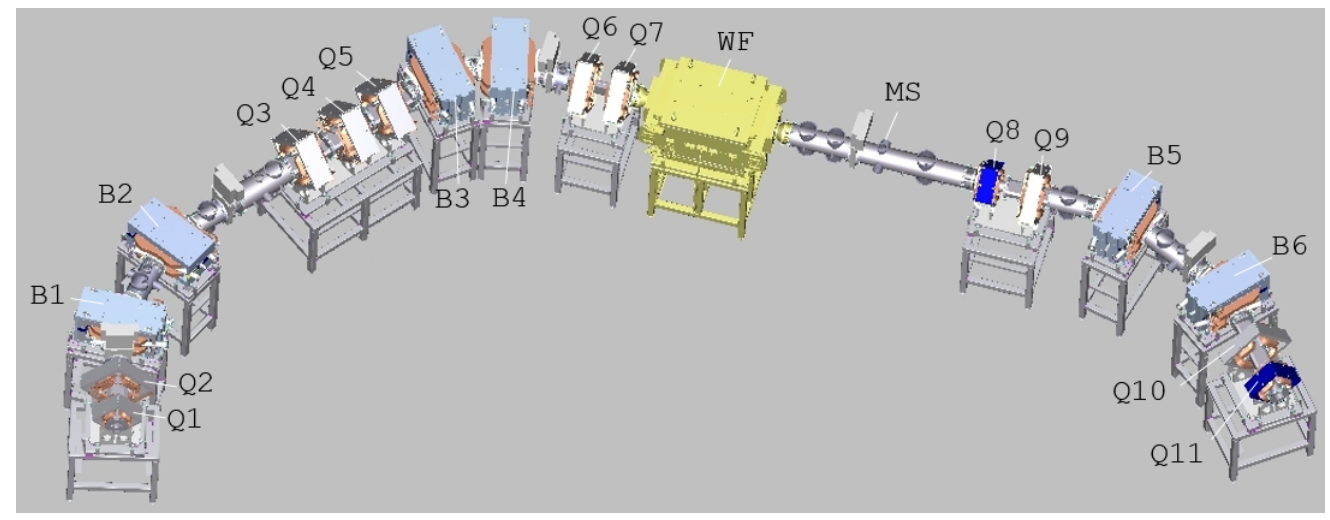

Figure 2: Schematic of the St. George recoil separator, where the labels correspond to "B" for dipole magnets, "Q" for quadrupole magnets, "WF" for the Wien filter, and "MS" for the mass slits. The target position is located upstream of Q1 and the recoil detection system (not shown) is downstream of Q11.

1.4. With fields on Q1 and Q2 set to provide a narrow 244 waist at the $\mathrm{B} 30^{\circ}$ exit-port quartz-viewer, the $\mathrm{B} 1_{245}$ and $\mathrm{B} 2$ fields were adjusted to minimize steering in the quadrupole magnets Q3-Q5. These adjust-246 ments were generally within $\lesssim 0.3 \%$ of the calculated ${ }^{247}$ optimal field setting. A tune with simultaneously ${ }^{248}$ non-steering Q1-Q2 and Q3-Q5 was not achievable, ${ }^{249}$ likely due to a minor misalignment of the quadrupole ${ }^{250}$ magnets. However, the shift of the beam spot posi- ${ }^{251}$ tion from steering was minimized to a vertical deflec- ${ }_{252}$ tion of $\lesssim 2.5 \mathrm{~mm}$ on the quartz viewer, which corre- ${ }^{252}$ sponds to a maximum deflection of $\lesssim 2$ mrad by the $e_{254}$ Q5 quadrupole magnet at maximum field strength. Given the Q5 field gradient, the observed deflection 255 indicates the vertical misalignment of the Q3-Q5256 quadrupoles is $\lesssim 1 \mathrm{~mm}$ with respect to the Q1-Q2. ${ }^{257}$

1.5. Quadrupole magnets Q1-Q5 and dipole magnets B3- ${ }^{258}$ B4 were adjusted to their nominal fields for the species of interest at the tune-energy, sending the beam to ${ }_{261}^{200}$ the B5 $0^{\circ}$ exit-port quartz-viewer, with no field on the B5 dipole. A small electric field was applied $t_{0}{ }^{262}$ the Wien filter to offset the residual magnetic field. ${ }^{263}$ The B3 and B4 fields were adjusted to achieve the ${ }_{265}$ non-steering condition on the B5 $0^{\circ}$ exit-port quartz ${ }^{265}$ viewer for the Q6-Q7 quadrupole magnets. $\quad{ }^{267}$

1.6. The Wien filter magnetic and electric fields, as well ${ }^{268}$ as the magnetic field clamps, were set to their nom- ${ }^{269}$ inal values for the species of interest at the tune-270 energy. The Wien filter magnetic field was then ad-271 justed to achieve the non-steering condition for the ${ }^{272}$ Q8-Q9 quadrupole magnets on the B5 $0^{\circ}$ exit-port $^{273}$ quartz-viewer.

1.7. The electrically insulated slits with charge readout s276 $_{27}$ located $1.59 \mathrm{~m}$ downstream of the Wien filter, the socalled 'mass slits', were then closed to the point of ${ }^{277}$ reading $\lesssim 1 \mathrm{pA}$ on the left and right mass slits. This ${ }^{278}$ provides a measurement of the beam width, which we ${ }^{279}$ can use to estimate the anticipated beam-rejection of the separator.

1.8. The B5 and B6 dipole magnets were then set to and adjusted about their nominal field settings for the species of interest at the tune-energy until the nonsteering condition was achieved for the quadrupole magnets Q10-Q11 on the quartz-viewer located on the $0^{\circ}$ exit port of the recoil detection chamber.

The second procedure, maximizing the energy acceptance of St. George, was accomplished by performing the following steps:

2.1. Transmission was checked by monitoring the beam current on a collimator upstream of the target location and monitoring the beam current on Faraday cups at the target and recoil detection locations, while extending and retracting the target location Faraday cup (See Figure 4.). For the calculated ion optical tune for the species of interest, we did not find $100 \%$ beam transmission from the target location to the recoil detector plane. Therefore, the quadrupole magnets Q1-Q11 required adjustment to achieve $100 \%$ transmission. With all other quadrupole magnets set to their nominal field setting, each quadrupole was individually adjusted in order to maximize the beam transmission to the recoil detector plane. Then, for each of the $N$ quadrupole magnets which improved beam transmission upon adjustment, the field was adjusted from the nominally calculated value by $1 / N$ times the individual adjustment required for that quadrupole magnet to improve beam transmission. This naïve scaling procedure resulted in $100 \%$ beam transmission (See Figure 4a.).

2.2. Having achieved $100 \%$ beam transmission for the tune-energy, the St. Ana terminal voltage was adjusted so that the beam would have an energy $-8 \%$ 


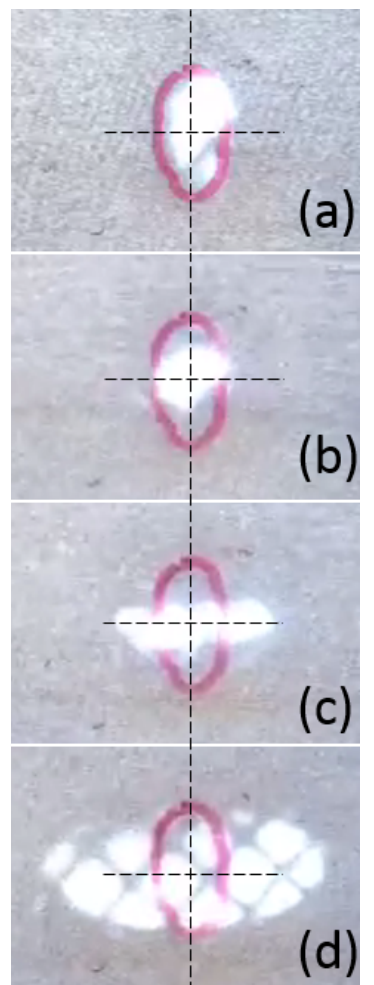

Figure 3: Demonstration of a non-steering ion optical tune. (a)- ${ }^{325}$ (d) show images of the B3 dipole magnet $0^{\circ}$ quartz viewer for $\mathrm{a}^{325}$ $1 \mathrm{MeV} \mathrm{H}^{+}$beam, where the Q2 quadrupole magnet is scaled from ${ }^{326}$ its minimum field strength (a) up to $\approx 0.13 \mathrm{~T}$, which is $\sim 50 \%$ of its 327 maximum value $(\mathrm{d})$, where $(\mathrm{b})$ and $(\mathrm{c})$ are at arbitrary intermediate $\mathrm{s}_{328}$ field strengths. The red oval and black crosses provide a reference for the size and center of the beam. The height and width of the red oval correspond to $\approx 3 \mathrm{~mm}$ and $\approx 1.5 \mathrm{~mm}$, respectively, on the quartz ${ }_{329}$ viewer.

below the tune-energy, e.g. to $1.84 \mathrm{MeV}$ from a 332 tune-energy of $2 \mathrm{MeV}$. In general, $100 \%$ transmis-3зз sion was not achieved. If beam current was lost on ${ }^{334}$ the mass slits, quadrupole magnets Q1-Q6 were ad-335 justed as described in the previous step to optimize beam transmission. Once beam current was removed from the mass slits, quadrupole magnets Q8-Q11 were adjusted as described in the previous step to optimize beam transmission.

2.3. Beam transmission was checked again with the accelerator adjusted back to the tune-energy. If $100 \%$ beam transmission was not achieved, the procedure from the previous step was repeated. This and the previous step were performed iteratively until $100 \%$ beam transmission was obtained for both the tuneenergy and the $-8 \% \frac{\Delta E}{E}$ energy.

2.4. Having achieved $100 \%$ transmission for the tuneenergy and $-8 \%$ below the tune-energy, the St. Ana terminal voltage was adjusted so that the beam would have an energy $+8 \%$ above the tune-energy, e.g. to $2.16 \mathrm{MeV}$ from a tune-energy of $2 \mathrm{MeV}$. If $100 \%$ transmission was not achieved, adjustments needed to be made to Q1-Q11. These adjustments, along with a re-verification of transmission at the tuneenergy and $-8 \%$ below the tune-energy, were performed in an iterative fashion, as described in the previous step. In general, we found the ion optical tune needed little to no adjustment to achieve $100 \%$ transmission at $+8 \%$ above the tune-energy once $100 \%$ transmission was achieved for the tuneenergy and $-8 \%$ below the tune-energy.

We note that the second of the two aforementioned procedures was greatly expedited for ensuing energy acceptance measurements, as the the suspected optimum values for the nominal tune were improved with time. For instance, for the third successful energy acceptance measurement only a single adjustment of quadrupole magnets Q10-Q11 was required in order to achieve $\pm 8 \%$ energy acceptance.

Figure 4 demonstrates $\pm 8 \%$ energy-acceptance for a $2 \mathrm{MeV}{ }^{16} \mathrm{O}^{2+}$ beam. The fluctuations observed in beam intensity are thought to be mainly due to instabilities in the St. Ana ECR ion source. The spikes in current for the Faraday cup at the target location following its insertion and retraction are thought to be due to current induced by the motion of the cup apparatus.

As seen in Figure 5, we observe full transmission to a precision of $\sim 1 \mathrm{nA}$ for a beam intensity of $\sim 100 \mathrm{nA}$, i.e. $>99 \%$

\section{Results and Discussion}

Energy acceptance measurements were performed as described in the previous section for 8 ion beams, which were chosen to span the phase space of $\mathrm{E} \rho$ and $\mathrm{B} \rho$ where recoil nuclei from $(\alpha, \gamma)$ reactions of astrophysical interest are anticipated. Table 1 lists the ion beams for which an energy acceptance measurement was performed.

Table 1: Ion beams for which $\pm 8 \%$ energy acceptance was verified for the St. George recoil separator. Uncertainties for kinetic energy $T$, magnetic rigidity $B \rho$, and electric rigidity $E \rho$ are $\approx 1 \%$, which is dominated by the present uncertainty of the analyzing magnet energy calibration.

\begin{tabular}{|c|c|c|c|c|c|}
\hline Species & Mass $[u]$ & Charge [e] & $\mathrm{T}[\mathrm{MeV}]$ & $\mathrm{B} \rho[\mathrm{Tm}]$ & $\mathrm{E} \rho[\mathrm{MV}]$ \\
\hline${ }^{16} \mathrm{O}^{4+}$ & 16 & 4 & 2.0 & 0.211 & 1.0 \\
\hline${ }^{1} \mathrm{H}^{1+}$ & 1 & 1 & 1.0 & 0.149 & 2.0 \\
\hline${ }^{4} \mathrm{He}^{1+}$ & 4 & 1 & 1.0 & 0.298 & 2.0 \\
\hline${ }^{20} \mathrm{Ne}^{4+}$ & 20 & 4 & 4.0 & 0.333 & 2.0 \\
\hline${ }^{16} \mathrm{O}^{2+}$ & 16 & 2 & 2.0 & 0.421 & 2.0 \\
\hline${ }^{4} \mathrm{He}^{2+}$ & 4 & 2 & 3.0 & 0.258 & 3.0 \\
\hline${ }^{16} \mathrm{O}^{4+}$ & 16 & 4 & 6.0 & 0.365 & 3.0 \\
\hline${ }^{16} \mathrm{O}^{4+}$ & 16 & 4 & 8.0 & 0.421 & 4.0 \\
\hline
\end{tabular}



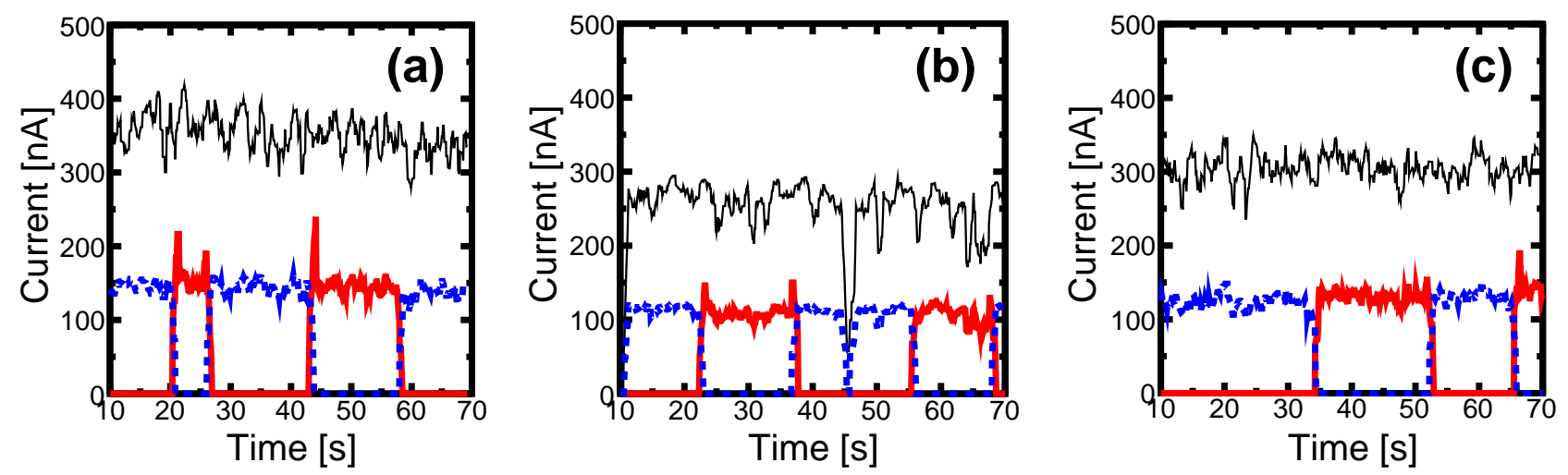

Figure 4: (color online.) Demonstration of $100 \%$ beam transmission through St. George for ${ }^{16} \mathrm{O}^{2+}$ ions with the recoil separator tuned for $\mathrm{B} \rho=0.421 \mathrm{Tm}, \mathrm{E} \rho=2.0 \mathrm{MV}(2 \mathrm{MeV}$ ions, in this case.). Figures (a), (b), and (c) show the beam current read at the target location (thick red lines), detector plane (thick blue-dashed lines), and collimator upstream of St. George used to monitor the incoming beam-current stability (thin black lines), for $2 \mathrm{MeV}$ (a), $2.16 \mathrm{MeV}$ (b), and $1.84 \mathrm{MeV}$ (c) ions.

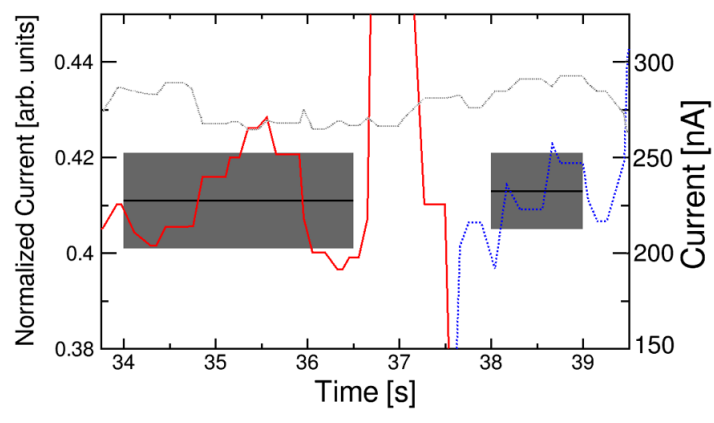

Figure 5: (color online.) Demonstration of $>99 \%$ beam transmission ${ }^{359}$ through St. George for $2.16 \mathrm{MeV}^{16} \mathrm{O}^{2+}$ ions with the recoil separator ${ }^{360}$ tuned for $\mathrm{B} \rho=0.421 \mathrm{Tm}, \mathrm{E} \rho=2.0 \mathrm{MV}$ ( $2 \mathrm{MeV}$ ions, in this case.). ${ }^{361}$ The ratio (left axis) of the current on the target-cup (red line) $\operatorname{and}_{362}$ detector cup (blue-dashed line) to the upstream collimator current are shown for a time of near-constant upstream collimator current (gray-dotted line, right axis). Two gray boxes indicate (1) horizon- ${ }^{364}$ tally: the time-frame during which the target cup is not moving, 365 in order to avoid unwanted current induced by the motion of the $\mathrm{e}_{366}$ cup and (2) vertically: the average current ratio (black line) and standard deviation of the current ratio (gray band). The mean current ratios differ by less than $1 \%$, indicating better than $99 \%$ beam $^{368}$ transmission.

\section{.} Furthermore, a direct measurement of ${ }^{22} \mathrm{Ne}(\alpha, \gamma)^{26} \mathrm{Mg}$ in
the energy range previously studied in regular kinemat-
ics [19] is well within reach, which would help constrain the role of the ${ }^{22} \mathrm{Ne}(\alpha, \mathrm{n}) s$-process neutron source. Finally, we demonstrate our ability to accept ${ }^{16} \mathrm{O}$ recoils from ${ }^{12} \mathrm{C}(\alpha, \gamma)$, noting that the large angular acceptance anticipated for St. George will allow the lowest-energy direct measurement of this reaction to date.

We used the measured width of the beam at the mass slits, as determined in Step 1.7, to provide a first-order estimate of the anticipated beam rejection capabilities of St. George. The smallest anticipated spatial separation between beam and recoil nuclei provided by the Wien filter is $\approx 5 \mathrm{~cm}$, which is for the case of an $(\alpha, \gamma)$ reaction on an $A=40$ nucleus. In order to achieve the desired mass rejection of 1 part in $10^{15}$, the magnitudes of the beam and recoil nuclei distributions must be similar at the location of the mass slits when the amplitude of the beam distribution is $10^{15}$ times larger than the amplitude of the recoil distribution. If we assume beam and recoil nuclei both have Gaussian horizontal distributions with the same width, but the beam distribution is $10^{15}$ larger in amplitude and has a centroid $5 \mathrm{~cm}$ horizontally displaced 


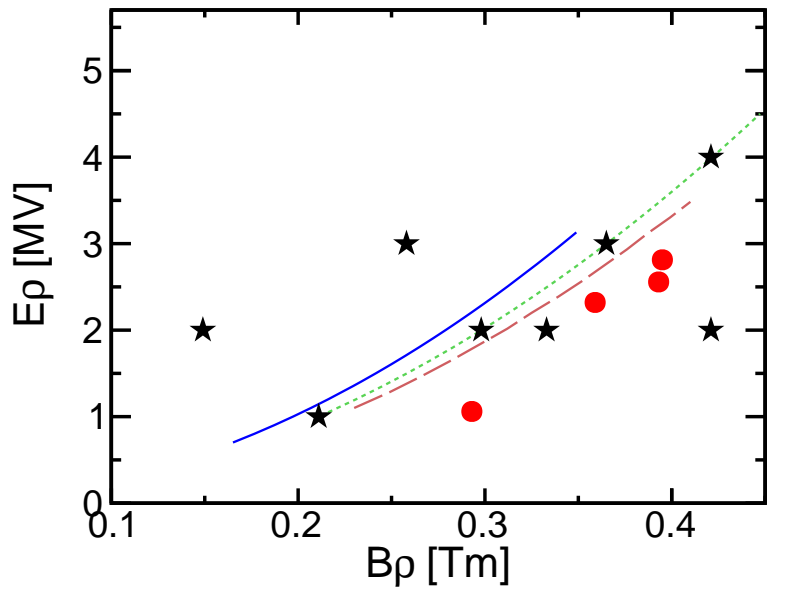

Figure 6: (color online.) Rigidity phase space designed to be ac-42 cepted by the St. George recoil separator, where the $\mathrm{B} \rho-\mathrm{E} \rho$ of ions with a measured energy acceptance are indicated with black stars. 422 Examples of recoils of astrophysical interest, low recoil-energy cases ${ }_{423}$ from Table 1 of Reference [10], assuming the most populous equilibrium charge-state from the dilute gas model of Reference [17] that is ${ }^{424}$ within the $\mathrm{B} \rho-\mathrm{E} \rho$ limits of St. George, are indicated by the red-filled 425 circles. The solid blue line indicates the $\mathrm{B} \rho-\mathrm{E} \rho$ space for $q=2^{+}$re$^{-}{ }_{42}$ coils from the ${ }^{3} \mathrm{He}(\alpha, \gamma)^{7}$ Be reaction at the energies measured in the ${ }_{427}$ past with the recoil separator ERNA [18]. The long-dashed mauve line corresponds to ${ }^{26} \mathrm{Mg}^{6+}$ recoils from the ${ }^{22} \mathrm{Ne}(\alpha, \gamma)$ reaction for energies covered by a previous measurement in forward kinematics [19]. The short-dashed green line corresponds to ${ }^{16} \mathrm{O}^{4+}$ recoils $^{428}$ from ${ }^{12} \mathrm{C}(\alpha, \gamma)$ for center of mass energies from $700-3000 \mathrm{keV}$.

from the recoil distribution centroid, a standard deviation ${ }_{433}$ of $\sigma \approx 4 \mathrm{~mm}$ for both distributions is sufficient to achieve ${ }^{434}$ a 1:1 ratio of beam-to-recoil nuclei $-3 \sigma$ left of the beam- ${ }^{435}$ line center at the mass slits $\left(3 \sigma\right.$ is chosen as the point ${ }_{437}^{436}$ for comparison since $\pm 3 \sigma$ encompasses $99.8 \%$ of the recoil ${ }_{438}$ distribution.). In Step 1.7 we determined the width of the ${ }^{439}$ ion distribution at the mass slits is $w \leq 20 \mathrm{~mm}$, which cor- ${ }^{440}$ responds to intercepting $\lesssim 1 \mathrm{pA}$ out of the full $\sim 100 \mathrm{nA}_{442}^{441}$ ion intensity (i.e. $\sim 0.001 \%$ ). For a Gaussian distribution, 443 this implies a mass slit opening of $\pm 4.5 \sigma$. Therefore, from ${ }^{444}$ our measurement of $w$, we deduce $\sigma \lesssim 2.2 \mathrm{~mm}$, which is ${ }_{446}^{445}$ far less than the $4 \mathrm{~mm}$ required for the desired mass re- ${ }_{447}$ jection. We note that our estimate is a best-case scenario448 since more pathological beam distributions are often ob- ${ }^{449}$ served $^{8}$. Furthermore, this estimate neglects effects such $_{451}^{450}$ as beam scattering within the separator which adversely ${ }_{452}^{451}$ impact the mass rejection.

\section{Conclusions}

We present first results from commissioning of the St. George recoil separator. By performing measurements of stable ion beams of varied elements, charge-states, and energies, we verify that St. George meets the design specifications of providing an energy acceptance of $\pm 8 \%$ within the separator's rigidity phase space. Our ultimately employed magnetic field strengths generally agree with predictions from ion-optical calculations performed with COSY Infinity; however, some deviations persist. We attribute present disagreements to the absence of realistic field maps in the calculations and an absence of an angular opening of the beam at the target location, which will be more restrictive when determining the optimum ion-optical tune. Our comparison to the properties of recoils from anticipated future nuclear reaction measurements demonstrates that St. George will be a vital tool used to obtain precise cross sections for $(\alpha, \gamma)$ reactions of astrophysical interest.

\section{Acknowledgements}

We thank the staff of the Nuclear Science Laboratory at the University of Notre Dame for their outstanding support. This material is based upon work supported by the National Science Foundation under Grants No. 1062819, 1419765, and 1430152, and the Nuclear Regulatory Commission under Grant No. NRC-HQ-12-G-38-0073.

\section{References}

[1] C. R. Brune, B. Davids, Ann. Rev. Nucl. Part. S. 65 (2015) 87.

[2] F. Käppeler, et al., Astrophys J. 437 (1994) 396.

[3] M. Wiescher, F. Käppeler, K. Langanke, Annu. Rev. Astron. Astr. 50 (2012) 165.

[4] C. Tur, A. Heger, S. Austin, Astrophys J. 671 (2007) 821.

[5] G. Magkotsios, F. X. Timmes, A. L. Hungerford, C. L. Fryer, P. A. Young, M. Wiescher, Astrophys J. Suppl. S. 191 (2010) 66.

[6] R. H. Cyburt, A. M. Amthor, A. Heger, E. Johnson, Z. Meisel, H. Schatz, K. Smith, Astrophys. J. 830 (2016) 55

[7] A. Caciolli, et al., Eur. Phys. J. A 39 (2009) 179.

[8] C. Ruiz, U. Greife, U. Hager, Eur. Phys. J. A 50 (2014) 99.

[9] C. Akers, et al., Phys. Rev. Lett. 110 (2013) 262502.

[10] M. Couder, G. Berg, J. Görres, P. LeBlanc, L. Lamm, E. Stech, M. Wiescher, J. Hinnefeld, Nucl. Instrum. Meth. A 587 (2008) 35.

[11] A. Kontos, et al., Nucl. Instrum. Meth. A 664 (2012) 272.

[12] Z. Meisel, K. Shi, A. Jemcov, M. Couder, Nucl. Instrum. Meth. A 828 (2016) 8.

[13] C. Matei, et al., Phys. Rev. Lett. 97 (2006) 242503.

[14] D. Rogalla, et al., Nucl. Instrum. Meth. A 513 (2003) 573.

[15] D. Rogalla, et al., Eur. Phys. J. A 6 (1999) 471.

[16] K. Makino, M. Berz, Nucl. Instrum. Meth. A 427 (1999) 338.

[17] R. O. Sayer, Rev. Phys. Appl. 12 (1977) 1543.

[18] A. Di Leva, et al., Phys. Rev. Lett. 102 (2009) 232502.

[19] K. Wolke, et al., Z. Phys. A 334 (1989) 491.

[20] E. G. Adelberger, et al., Rev. Mod. Phys. 83 (2011) 195.

\footnotetext{
${ }^{8}$ This has been observed by an in-beam fluorescing quartz viewer located after the mass slits, which was added to the set-up following the measurements described in this work.
} 